\title{
An update on paediatric respiratory diseases
}

\author{
Ralph Epaud ${ }^{1,2,3,4,5}$
}

Affiliations: ${ }^{1}$ Pediatric Dept, Centre Intercommunal de Créteil, Créteil, France. ${ }^{2}$ Inserm, Unité 955, Equipe 5, Créteil, France. ${ }^{3} \mathrm{DHU}$ Ageing Thorax Vessel Blood, Créteil, France. ${ }^{4}$ Centre des Maladies Respiratoires Rare, Respirare ${ }^{\circledR}$, Paris, France. ${ }^{5}$ Université Paris-Est, Faculté de Médecine, Créteil, France.

Correspondence: Ralph Epaud, Pediatric Dept, Centre Intercommunal de Créteil, 40 av de Verdun, 94000 Créteil, France. E-mail: ralph.epaudachicreteil.fr

@ERSpublications

An update of paediatric lung diseases including interstitial lung disease, virus causing wheezing disorders and diaphragmatic hernia together with new imaging diagnosis tools http://ow.ly/TclX30iZazK

Cite this article as: Epaud R. An update on paediatric respiratory diseases. Eur Respir Rev 2018; 27: 180013 [https://doi.org/10.1183/16000617.0013-2018].

During the 2017 European Respiratory Society International Congress in Milan, Italy, exciting topics on childhood lung disease were reviewed in the session "Paediatric year in review: update on paediatric pulmonology". These brief reviews highlighted publications in the field of paediatric lung disease and were presented by well-known experts within the field.

The first review by Matthias Griese focused on chronic interstitial lung disease in children [1]. In the past decade, growing attention has been given to rare paediatric lung diseases. One of the areas of interest is childhood interstitial lung disease (ChILD), a rare diagnosis comprising more than 200 different lung conditions [2]. The reported disease prevalence is 0.36 cases per 100000 children [3]. Given this low incidence, inter-individual comparison of the onset, severity and disease course is limited. Recently, comprehensive classification systems [4] and clinical networks [2], such as the Center of Paediatric Lung Disease, launched in France in 2006, were developed. However, we still lack consensus on the standardised assessment of this disease and multicentric collaborations are the only method of successful clinical research in ChILD. To address this problem, the European Union Framework Programme FP7 grant "ChILD - European Management Platform" (www.childeu.net) aims at building a pan-European consensus for standardised diagnosis and treatment protocols in ChILD [2]. During the funding period, thanks to the different ChILD centres in Europe, significant insight was made into current clinical standards for diagnosis, therapeutic strategies, follow-up and management of exacerbations. M. Griese reported recent updates on persistent tachypnoea of infancy, a rare condition that remains frequently undiagnosed because of a variable and often relatively mild phenotype. This presentation should be differentiated from chronic forms of inherited surfactant-dysfunction disorders such as NKX2-1, SFTPC or ABCA-3 mutation-associated disorders, which probably represent $>25 \%$ of the cases. Studies collecting cases have improved our knowledge on these genetic diseases and their response to usual ChILD treatment, including steroids, hydroxychloroquine and azithromycin, as well as their outcomes [5, 6]. For example, a study of children with SFTPC mutations showed significantly improved respiratory conditions with early treatment in most children, whereas 15 years ago, most of these patients would have had lung transplantation [6]. The review by M. Griese highlighted a follow-up of a cohort of patients with inherited surfactant disorders and a more severe phenotype who underwent lung transplantation [1]. Recently, exome sequencing of undiagnosed family cases identified a new inherited auto-inflammatory syndrome

Received: Feb 102018 | Accepted: March 152018

Provenance: Commissioned article, peer reviewed.

Copyright OERS 2018. ERR articles are open access and distributed under the terms of the Creative Commons Attribution Non-Commercial Licence 4.0. 
caused by mutations in the transmembrane protein 173 (TMEM173) responsible for systemic inflammation, cutaneous vasculopathy and pulmonary inflammation, which were also mentioned in this review. All this progress in disease diagnosis and treatment was made possible by the combined experience of the different European teams. M. Griese pointed out the need to continue collecting cases and biomaterials to study the natural course, molecular disease causes, appropriate diagnostic steps, treatment options and meaningful prophylaxis.

The second review by Peter Le Souëf [7] focused on viral infections in wheezing disorders. The growing literature has shown the important role of viral infections in early ages, which in turn may predispose to the development of chronic lung diseases such as asthma in children and chronic obstructive pulmonary disease (COPD) in adults [8]. These studies contradict the prevailing idea that patients destined to have no lung disease and those destined to have COPD initially reach the same plateau for lung function and evolve further whether they are cigarette smokers or not. More recently, several papers provided evidence that lower respiratory tract illnesses (especially pneumonia before 3 years of age) may alter lung function during the preschool years, which further continues to decline at a slower pace thereafter [8]. In this context, we need studies examining the mechanisms by which respiratory viruses impair lung function at a young age and the effects of novel interventions to reduce this aggression. P. Le Soueff presented several papers that aimed to decipher the mechanism of viral infections, with a focus on rhinovirus and its effects on innate immunity. Results of these studies are important for developing new therapeutics for both the treatment and prevention of small airway alterations. Yet, the review also reported papers assessing the impact of steroids, an older treatment, and omalizulab, a more recent one, on the development of asthma. With the development of multiplex metagenomic analyses by use of next-generation sequencing, we know now that the diversity of viral and bacterial colonisation in the upper and lower airways is greater than previously revealed by routine tool analysis. Using these new techniques, several studies aimed to assess the interaction between viruses and bacteria in preschool lung infection. Importantly, they suggest that the virome appears to be diverse in children with a symptomatic infection, which contrasts with previous results on the bacterial microbiome showing reduced diversity in children with a respiratory disease.

In a third review Daphne S. Mous [9] presented the most recent papers exploring the molecular pathway that connects diaphragmatic hernia, pulmonary lung hypertension and lung hypoplasia. Congenital diaphragmatic hernia $(\mathrm{CDH})$ is a rare condition that occurs when the diaphragm does not develop normally, which is responsible for lung disease with extremely high morbidity and mortality. A hole in the diaphragm allows the organs in the abdomen to move into the chest cavity, thus pushing out the heart and lung and limiting proper lung development. One of the most important issues in $\mathrm{CDH}$ is the degree of lung hypoplasia, which depends to a large extent on when the hernia appears during pregnancy [10]. This condition illustrates the close interaction between lung vessel and airways and the pressure stresses applied on the lung during pregnancy, but also how we must improve our comprehension of the disease to be able to develop new therapeutics. Important developments in endothelin and the retinoic acid signalling pathway, as well as the role of the pericyte, a perivascular cell that stabilises the newly formed endothelial tubes very early in lung development, were provided in the review by D.S. Mous. Among the different treatments reviewed, the most impressive is certainly fetoscopic endoluminal tracheal occlusion (FETO), whereby a small balloon is inserted in the fetal trachea to temporarily block the airway for 4 to 6 weeks, thereby resulting in higher internal pressure which forces the lung to grow. It is fascinating that the idea of blocking the airway emerged from the observation of an over-developing lung in patients with congenital high airway obstruction syndrome. Clinical trials investigating the role of FETO were performed in Belgium and France, with good results but a high price to pay in terms of perioperative mortality and morbidity [11].

With the huge progress in understanding paediatric lung disease together with the development of new therapies, we must improve our tools for diagnosis and disease assessment. This was the subject of the fourth review by Harm A.W.M. Tiddens [12] who addressed advances in lung imaging for children. Imaging is a must-have tool for diagnosis but also for following pulmonary disease. Huge progress has been made since the discovery of the chest radiograph in 1895 by the German physicist Wilhelm Röntgen through to the discovery of computed tomography (CT) in 1971 and magnetic resonance imaging in 1981. For many years, the presence of high volume of gas (air) in the lung has led to the preference for a CT scan for lung imaging. Recently, the "as low as reasonably achievable" concept in paediatric CT [13], i.e. inappropriate use and unnecessary radiation dosage should be avoided in this population, has led to questioning of the use of high-resolution CT scan for diagnosis and follow-up of children with chronic lung diseases. H.A.W.M. Tiddens presented recent papers exploring the relationship between high-resolution CT and pulmonary function. Although this approach has been studied in adult patients, it remains controversial in children, and interesting results in spirometry-guided CT for cystic fibrosis are presented. Recent studies aiming to develop methods to standardise image quality for CT and magnetic 
resonance imaging or explore lung infection and inflammation were included in this review and may contribute to improving the diagnostic yield in everyday clinical practice.

After reading these reviews, readers will be aware of the important progress made in the understanding, diagnosis and management of childhood lung diseases in 2017. Enjoy the lecture!

Conflict of interest: None declared.

\section{References}

Griese M. Chronic interstitial lung disease in children. Eur Respir Rev 2018; 27: 170100.

Bush A, Anthony G, Barbato A, et al. Research in progress: put the orphanage out of business. Thorax 2013; 68: 971-973.

3 Dinwiddie R, Sharief N, Crawford O. Idiopathic interstitial pneumonitis in children: a national survey in the United Kingdom and Ireland. Pediatr Pulmonol 2002; 34: 23-29.

4 Kurland G, Deterding RR, Hagood JS, et al. An official American Thoracic Society clinical practice guideline: classification, evaluation, and management of childhood interstitial lung disease in infancy. Am J Respir Crit Care Med 2013; 188: 376-394.

5 Nattes E, Lejeune S, Carsin A, et al. Heterogeneity of lung disease associated with NK2 homeobox 1 mutations. Respir Med 2017; 129: 16-23.

6 Thouvenin G, Abou Taam R, Flamein F, et al. Characteristics of disorders associated with genetic mutations of surfactant protein C. Arch Dis Child 2010; 95: 449-454.

Le Souëf P. Viral infections in wheezing disorders. Eur Respir Rev 2018; 27: 170133.

Martinez FD. Early-life origins of chronic obstructive pulmonary disease. N Engl J Med 2016; 375: 871-878.

Mous DS, Kool HM, Wijnen R, et al. Pulmonary vascular development in congenital diaphragmatic hernia. Eur Respir Rev 2018; 27: 170104.

10 Coleman A, Phithakwatchara N, Shaaban A, et al. Fetal lung growth represented by longitudinal changes in MRI-derived fetal lung volume parameters predicts survival in isolated left-sided congenital diaphragmatic hernia. Prenat Diagn 2015; 35: 160-166.

11 Jani JC, Nicolaides KH, Gratacos E, et al. Severe diaphragmatic hernia treated by fetal endoscopic tracheal occlusion. Ultrasound Obstet Gynecol 2009; 34: 304-310.

12 Tiddens HAWM, Kuo W, van Straten M, et al. Paediatric lung imaging: the times they are a-changin'. Eur Respir Rev 2018; 27: 170097.

13 The ALARA (as low as reasonably achievable) concept in pediatric CT intelligent dose reduction. Multidisciplinary conference organized by the Society of Pediatric Radiology. August 18-19, 2001. Pediatr Radiol 2002; 32: 217-313. 